\title{
Severe depression more common in patients with ductal carcinoma in situ than early-stage invasive breast cancer patients
}

\author{
M. L. Gregorowitsch ${ }^{1}$ - H. J. G. D. van den Bongard ${ }^{1}$ - D. A. Young-Afat ${ }^{1,2}$ \\ J. P. Pignol ${ }^{4}$ - C. H. van Gils ${ }^{2}$ A. M. May $^{2} \cdot$ H. M. Verkooijen ${ }^{3}$
}

Received: 29 August 2017/ Accepted: 1 September 2017/Published online: 11 September 2017

(c) The Author(s) 2017. This article is an open access publication

\begin{abstract}
Purpose Ductal carcinoma in situ (DCIS) is associated with an excellent prognosis; historical studies have shown similar levels of psychological distress in patients with DCIS and with early-stage invasive breast cancer (earlyIBC). It is suggested that these results might have led to better patient education about prognosis after DCIS. This study reports the current levels of anxiety, depression, and health-related quality of life (HRQoL) in DCIS and earlyIBC patients.

Methods DCIS $(n=89)$ and early-IBC patients, T1-2N0, $(n=361)$ were selected from the UMBRELLA breast cancer cohort. Patient-reported outcomes were prospectively collected before the start of adjuvant radiotherapy (baseline) and at 3, 6, 12, 18, and 24 months thereafter. Mixed models were used to compare differences in levels of anxiety, depression, and HRQoL between DCIS and early-IBC patients.
\end{abstract}

Electronic supplementary material The online version of this article (doi:10.1007/s10549-017-4495-y) contains supplementary material, which is available to authorized users.

M. L. Gregorowitsch

m.l.gregorowitsch@umcutrecht.nl

1 Department of Radiation Oncology, University Medical Center Utrecht, Heidelberglaan 100, 3584 CX Utrecht, The Netherlands

2 Department of Clinical Epidemiology, Julius Center for Health Sciences and Primary Care, University Medical Center Utrecht, Utrecht, The Netherlands

3 Imaging Division, University Medical Center Utrecht, Utrecht, The Netherlands

4 Department of Radiation Oncology, Erasmus Medical Center, Rotterdam, The Netherlands
Results DCIS and early-IBC patients reported similar levels of anxiety, which were highest at baseline. Depression scores were comparable between groups, also after stratification by use of adjuvant chemotherapy. The proportion of patients reporting high-risk depression scores (i.e., Hospital Anxiety and Depression Sale score $\geq 8$ ) was significantly higher among patients with DCIS at 6,12 and 18 months, and this proportion increased over the first 18 months. Health-related quality of life was comparable between both groups.

Conclusion Severe depression scores are more common in DCIS patients, despite their excellent prognosis. These results suggest that further improvement of patient education and effective patient doctor communication about the prognostic differences between patients with DCIS and invasive breast cancer is still highly needed.

\section{Introduction}

Ductal carcinoma in situ (DCIS) and early-stage invasive breast cancer (early-IBC) are diagnosed with greater frequency since the implementation of breast cancer-screening programs $[1,2]$. DCIS, which accounts for over $20 \%$ of new breast cancer diagnoses in the United States [3-5], is a non-invasive condition characterized by neoplastic cells within the breast ducts, with no theoretic potential for metastatic spread. With adequate treatment, 10-year breast cancer-specific mortality following DCIS is very low (<less than 2\%) [6-8], while 10-year breast cancer-specific mortality for early-IBC is $2-11 \%$ [9], resulting in a rapidly growing population of DCIS survivors.

Despite the lower mortality rate, treatment of DCIS resembles local treatment of early-IBC. In most Western 
countries, the majority of DCIS patients undergo breastconserving surgery with adjuvant whole breast irradiation. In contrast to patients with early-IBC, axillary staging (i.e., sentinel lymph node biopsy) is not indicated in patients with DCIS [7, 10].

Historically, DCIS patients have consistently reported similar levels of fear of breast cancer recurrence and death from breast cancer as women with early-IBC, which can cause substantial psychological distress [11-17]. Today, the excellent survival of DCIS is extensively discussed with the patient by the multidisciplinary team, and innumerable online documentation is available to patients on this matter. It is therefore expected that with the current knowledge about these misconceptions in DCIS patients, and with better information about the prognosis, DCIS patients would report today lower anxiety and depression than patients with early-IBC. However, no recent studies have been published comparing patient-reported outcomes from these groups, receiving modern day treatment in this era of better patient education.

The aim of this prospective cohort study was to evaluate the current status of self-reported anxiety, depression, and health-related quality of life (HRQoL) in the first 2 years after diagnosis in patients with DCIS and those with earlyIBC receiving treatment between 2013 and 2016.

\section{Methods}

This study was conducted within the prospective Utrecht cohort for Multiple BREast cancer intervention studies and Long-term evaLuAtion (UMBRELLA) [18]. UMBRELLA includes patients with invasive breast cancer and patients with DCIS, referred to the Department of Radiation Oncology of the University Medical Center (UMC) Utrecht for adjuvant radiotherapy. All participants were at least 18 years old, and were able to understand the Dutch language in written and spoken form. All participants gave informed consent for the collection of baseline demographics, tumor and treatment characteristics, clinical follow-up data, and patient-reported outcome measures (PROs) at regular time intervals. This study complies with the Dutch law on Medical Research in Humans and was approved by the Medical Ethical Committee of the UMC Utrecht, the Netherlands.

For the present study, we selected patients with histologically confirmed early-IBC or DCIS enrolled in UMBRELLA between October 2013 and February 2016. All patients with a minimal follow-up of 6 months who completed at least one follow-up questionnaire were eligible. Patients with recurrent breast cancer after enrollment were excluded. Tumor type was categorized either as isolated DCIS without micro-invasion or invasive breast cancer (i.e., invasive lobular, invasive ductal carcinoma, or DCIS with micro-invasion). The definition for early-IBC was a clinical and pathological tumor stage (TNM stage) of $\mathrm{T} 1$ or T2 without nodal involvement after axillary staging of N0 (i.e., negative sentinel node or negative axillary lymph node dissection).

All patients underwent breast cancer treatment by either undergoing mastectomy or breast-conserving surgery followed by whole breast irradiation varying from minimal 16 fractions of $2.66 \mathrm{~Gy}, 21$ or 23 fractions of 2.17 or $2.03 \mathrm{~Gy}$ with simultaneously integrated 0.49 or 0.63 Gy boost, respectively. Depending on patient and tumor characteristics (e.g., young age, tumor size, tumor grade, estrogen receptor status, HER2 receptor status), patients with earlyIBC were treated with chemotherapy, immunotherapy, and/ or hormonal therapy to minimize the risk of recurrence.

Clinical data were obtained from the Netherlands Cancer Registry (NCR), part of the Netherlands Comprehensive Cancer Organization (IKNL), which prospectively registers clinicopathological and treatment characteristics[19]. Data on PROs were collected through self-reported questionnaires administered at regular time intervals and registered within the Patient-Reported Outcomes Following Initial treatment and Long-term Evaluation of Survivorship (PROFILES)-registry [20]. PROs were collected before the start of adjuvant radiotherapy (baseline) and at 3, 6, 12, 18, and 24 months thereafter.

Anxiety and depression were assessed using the Hospital Anxiety and Depression Scale (HADS) [21, 22]. HADS is a 14-item self-rating scale mainly developed to measure the severity of symptoms of anxiety and depression in nonpsychiatric patients [22]. Items are grouped into two subscales, i.e., the HADS anxiety sub-scale and HADS depression sub-scale with a range of scores from 0 to 21 in each sub-scale. Lower scores on the anxiety- and depression sub-scale represent less symptoms. Based on validation, cut-off scores were used to categorize anxiety and depressive symptoms, values of $<8$ indicated standard levels of anxiety and depression. With a score of $8-10$, patients were considered to have possible anxiety and depression, while values greater than 10 indicate a high likelihood of anxiety or depressive disorders [21-23].

Health-related quality of life (HRQoL) was assessed using the European Organization for Research and Treatment of Cancer Quality of Life Questionnaire (EORTC QLQ-C30) [24]. Scores were generated in accordance with EORTC QLQ-C30 guidelines [25].

Anxiety and depression and HRQoL scores of DCIS and early-IBC patients were compared to an age-matched female Dutch reference population without a history of breast cancer [20]. 


\section{Statistical analysis}

Categorical data were summarized using frequencies and percentages. Continuous data were summarized using means and standard deviations. The Chi-Square test was used to compare differences in proportions between DCIS patients and patients with early-IBC.

To compare anxiety and depression scores and HRQoL between the two groups over time, linear mixed models for repeated measures were used, which take into account the correlation between the measurements within subjects. An autoregressive covariance structure was used for the mixed model to account for the correlations among observations, supposing that correlations are higher between measurements that were closer together than time-points further apart (i.e., decline exponentially) [26]. We included a random intercept per patient, which takes baseline PRO differences among patients into account. As fixed effects we entered time since enrollment (categorical), group (early-IBC and DCIS), age (continuous), and the interaction between time and group. Between-group effects were modeled using outcome measurements of anxiety and depression obtained at baseline and at $6,12,18$, and 24 months. For HRQoL, an additional measurement was taken at 3 months. Results were presented as estimated marginal means and mean differences (MD). We performed a stratified mixed model analysis to compare patients with DCIS and those with early-IBC patients without systemic treatment. All statistical tests were 2-sided and performed at a significance level of 0.05 . Statistical analyses were performed with Statistical Package for Social Sciences (SPSS) software (IBM SPSS Statistics for Windows, Armonk, NY: IBM Corp.)

\section{Results}

In total, 522 patients with primary DCIS or early-IBC were enrolled in UMBRELLA between October 2013 and February 2016 (Fig. 1). Responders to at least one followup questionnaire were selected for further analysis $(86 \%$, $n=450$ ). Responders did not differ from non-responders $(14 \%, n=72)$ with respect to age, pathological tumor stage, axillary surgery, and type of adjuvant radiotherapy (Supplement Data Table 1). Non-responders were more often treated with systemic treatment.

From the 450 respondents, 89 (20\%) patients were diagnosed with DCIS and $361(80 \%)$ patients with earlyIBC (Table 1). Mean age at enrollment in UMBRELLA was similar for both groups (59 years). The majority of patients $(72 \%, n=64$ for DCIS vs. $57 \%, n=206$ for early-IBC) were detected in the context of the national breast cancer-screening program. In both groups, almost all

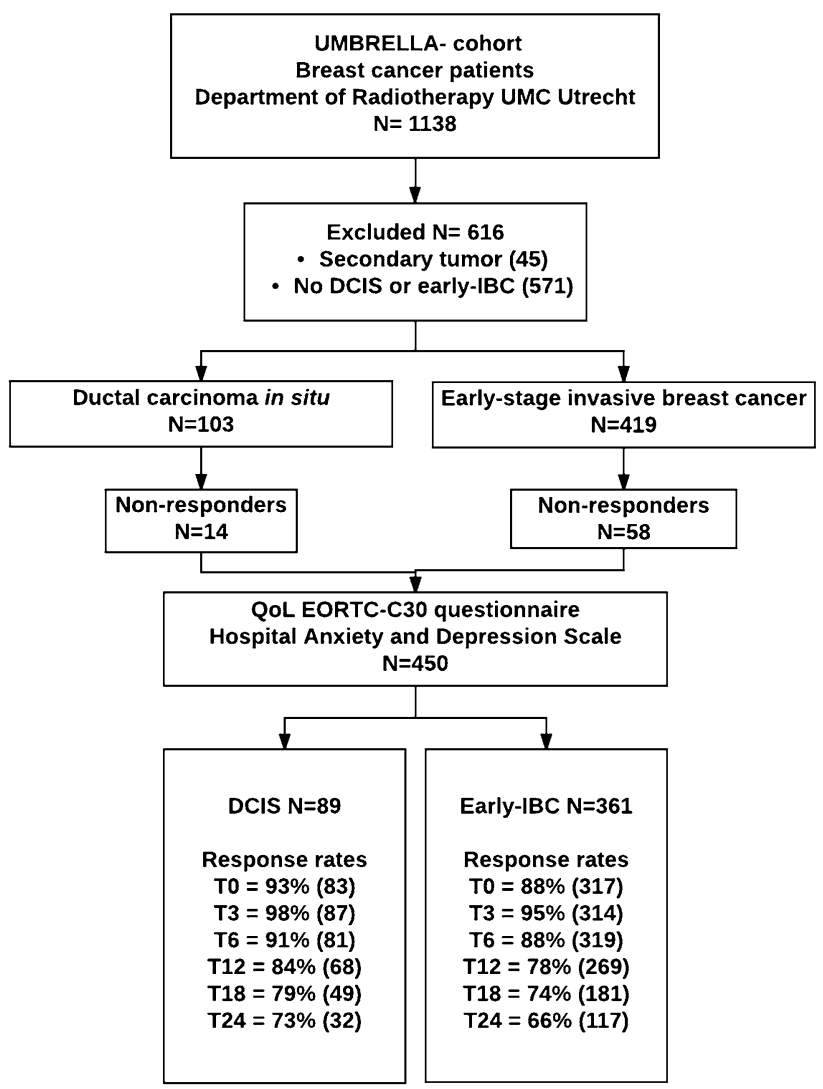

Fig. 1 Flowchart of patients inclusion within the UMBRELLA breast cancer cohort and questionnaire response rates

patients were treated with breast-conserving surgery (99\%, $n=88$ for DCIS; $98 \%, n=352$ for early-IBC). Whole breast irradiation consisted of 21 fractions with integrated boost to the tumor bed for the majority of DCIS patients $(69 \%, n=61)$, while the majority of early-IBC patients $(59 \%, n=213)$ received 16 radiation fractions on the breast or chest wall without an integrated radiation boost.

In the group of early-IBC, 286 patients (79\%) had T1 tumor (microscopic tumor measuring $2 \mathrm{~cm}$ or less), 32 patients $(8 \%)$ received neo-adjuvant systemic treatment, and 175 (49\%) received adjuvant systemic treatment.

For early-IBC patients, mean anxiety and depression scores slightly decreased over time, while mean anxiety scores for DCIS patients remained relatively stable up to the first 18 months of follow-up. In the first 12 months of follow-up the mean depression score reported by DCIS patients increased. There were no significant differences in anxiety and depression scores between DCIS and earlyIBC patients at any time-point (i.e., no significant interaction between time and group (DCIS or early-IBC) in any of the models). This pattern remained similar after stratification for systemic therapy (Fig. 2). For patients with DCIS a somewhat lower, but non-significant, anxiety score was observed at 24 months. On the depression sub-scale, 
Table 1 Demographics and disease characteristics of respondents participating in the UMBRELLA cohort

\begin{tabular}{|c|c|c|}
\hline & DCIS, $n(\%)$ & Early-IBC, $n(\%)$ \\
\hline Total no. of patients & $89(20)$ & $361(80)$ \\
\hline Age at inclusion, mean (SD) & $59(8)$ & $59(10)$ \\
\hline Breast cancer detected by screening (yes) & $64(72)$ & $206(57)$ \\
\hline \multicolumn{3}{|l|}{ Pathological tumor stage } \\
\hline DCIS & $89(100)$ & $2(1)$ \\
\hline $\mathrm{T} 1$ & $0(0)$ & $286(79)$ \\
\hline $\mathrm{T} 2$ & $0(0)$ & $54(15)$ \\
\hline $\mathrm{Tx}$ & $0(0)$ & $19(5)$ \\
\hline \multicolumn{3}{|l|}{ Invasive tumor grade } \\
\hline Grade I & $12(14)$ & $105(29)$ \\
\hline Grade II & $35(39)$ & $149(41)$ \\
\hline Grade III & $36(40)$ & $72(20)$ \\
\hline Unknown & $6(7)$ & $35(10)$ \\
\hline \multicolumn{3}{|l|}{ Estrogen receptor status } \\
\hline Negative & ND & $54(15)$ \\
\hline Positive $^{\mathrm{a}}$ & ND & $302(84)$ \\
\hline Unknown & NA & $5(1)$ \\
\hline \multicolumn{3}{|l|}{ HER2 receptor status } \\
\hline Negative & ND & $314(87)$ \\
\hline Positive & ND & $38(11)$ \\
\hline Unknown & NA & $9(2)$ \\
\hline \multicolumn{3}{|l|}{ Neo-adjuvant systemic treatment } \\
\hline None & $89(100)$ & $329(92)$ \\
\hline Chemotherapy and/or immunotherapy & $0(0)$ & $32(8)$ \\
\hline Unknown & $0(0)$ & $0(0)$ \\
\hline \multicolumn{3}{|l|}{ Surgical treatment } \\
\hline Breast-conserving surgery & $88(99)$ & $352(98)$ \\
\hline Mastectomy & $0(0)$ & $9(2)$ \\
\hline Other $^{\mathrm{b}}$ & $1(1)$ & $0(0)$ \\
\hline \multicolumn{3}{|l|}{ Axillary procedure } \\
\hline No axillary procedure & $29(33)$ & $0(0)$ \\
\hline Sentinel node biopsy & $60(67)$ & $355(98)$ \\
\hline Axillary lymph node dissection & $0(0)$ & $6(2)$ \\
\hline \multicolumn{3}{|l|}{ Adjuvant systemic treatment } \\
\hline None & $80(90)$ & $186(52)$ \\
\hline Chemotherapy and/or immunotherapy & $0(0)$ & $30(8)$ \\
\hline Chemotherapy and/or hormonal therapy & $0(0)$ & $46(13)$ \\
\hline Hormonal treatment & $0(0)$ & $98(27)$ \\
\hline Unknown & $9(10)$ & $1(0)$ \\
\hline \multicolumn{3}{|l|}{ Radiotherapy treatment } \\
\hline Local & $26(29)$ & $213(59)$ \\
\hline Local with boost tumor bed & $61(69)$ & $144(40)$ \\
\hline Locoregional $^{\mathrm{c}} \pm$ boost tumor bed & $0(0)$ & $2(1)$ \\
\hline Unknown & $2(2)$ & $2(1)$ \\
\hline
\end{tabular}


Table 1 continued

\begin{tabular}{lll}
\hline & DCIS, $n(\%)$ & Early-IBC, $n(\%)$ \\
\hline Median follow-up in months (IQR) & $18 ; 15$ & $18 ; 12$ \\
\hline
\end{tabular}

Categories may not sum to total $N$ because of missing values. Data may not total to $100 \%$ because of rounding

$D C I S$ ductal carcinoma in situ, Early- $I B C$ early-stage invasive breast cancer, $S D$ standard deviation, $N D$, not determined, $N A$ not applicable, HER2 human epidermal growth factor receptor 2, IQR interquartile range

${ }^{a}$ Estrogen receptor positive $>10 \%$

${ }^{\mathrm{b}}$ Tumor was removed during breast reduction

${ }^{\mathrm{c}}$ Radiotherapy on the breast/chest wall and on the axilla levels I-II or I/II-IV
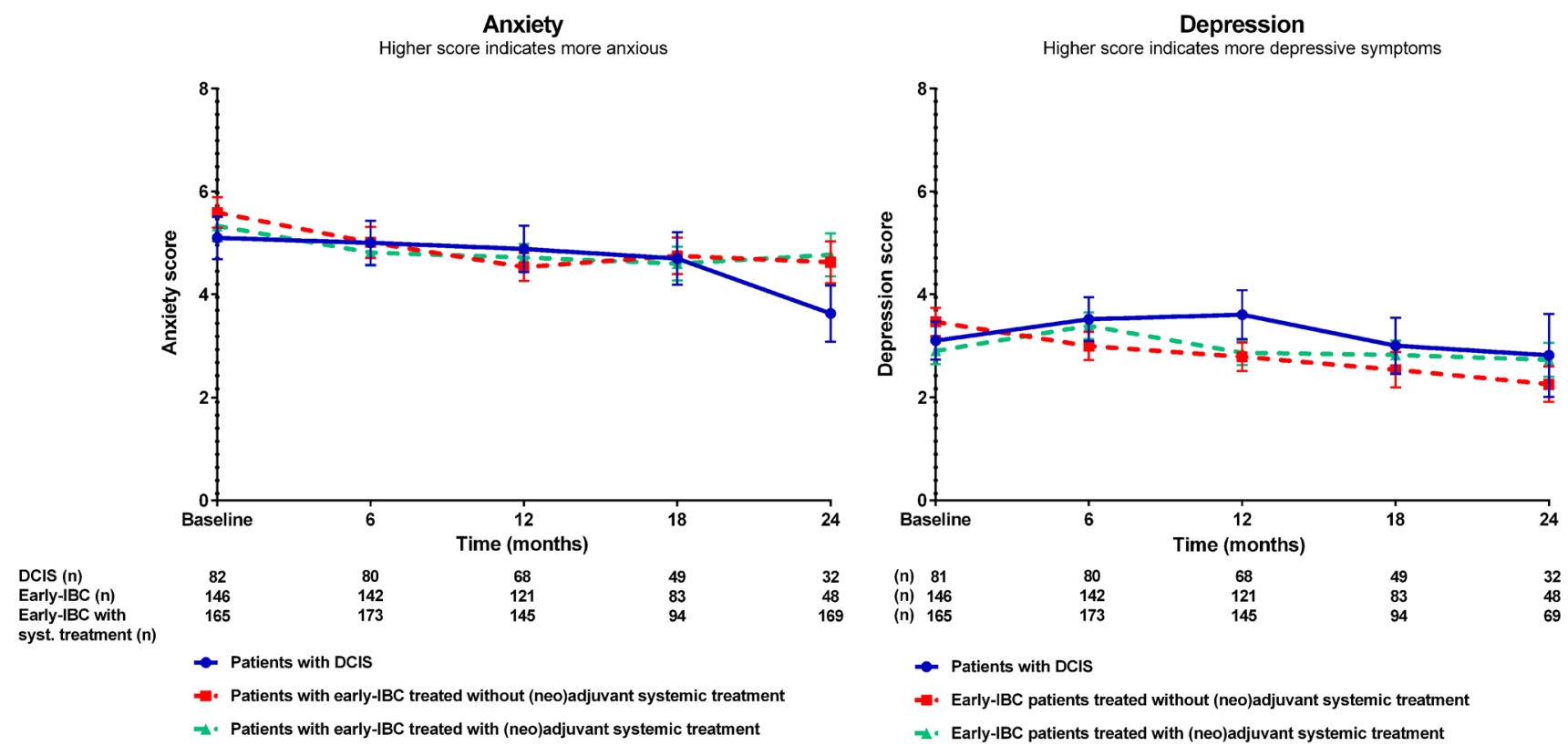

Fig. 2 Mean scores on the Hospital Anxiety and Depression Scale (HADS) of patients with ductal carcinoma in situ, patients with early invasive breast cancer without (neo)adjuvant systemic treatment and

patients with early invasive breast cancer treated with (neo)adjuvant systemic treatment at each time-point

patients with DCIS report slightly higher scores at 6,12 , 18, and 24 months (Table 2), but these differences were not significant.

Adjusted HADS scores (for age) from mixed model analysis were similar for early-IBC patients treated without systemic treatment and DCIS patients, indicating that anxiety and depression scores were similar between DCIS and early-IBC patients, irrespective of systemic treatment (Supplement data Table 2).

The proportion of patients reporting anxiety scores $\geq 8$, indicating an increased likelihood of anxiety disorder, was not significantly different between DCIS and early-IBC patients (Fig. 3). For both groups, high-risk anxiety scores decreased over time. The proportion of patients with DCIS reporting high depression scores $(\geq 8)$ was significant higher than the proportion of patients with early-IBC at 6 ,

12, and 18 months. For patients with DCIS, the proportion reporting high-risk depression scores increased within the first 18 months, in contrast to patients with early-IBC where a decrease in high-risk scores was observed over time. Compared to the normative population, the proportion of patients reporting high-risk anxiety scores was higher in both groups in the first 18 months. The proportion of DCIS patients with high-risk depression scores at 6,12 , 18 , and 24 months was higher compared to the normative population in contrast to the proportion of early-IBC patients.

Health-related quality of life scores (global health) were comparable between DCIS and early-IBC patients (Supplement data Table 3). Scores were slightly lower during treatment (at 3 months), improved at 6 months, and increased above baseline level as of 12 months in both 


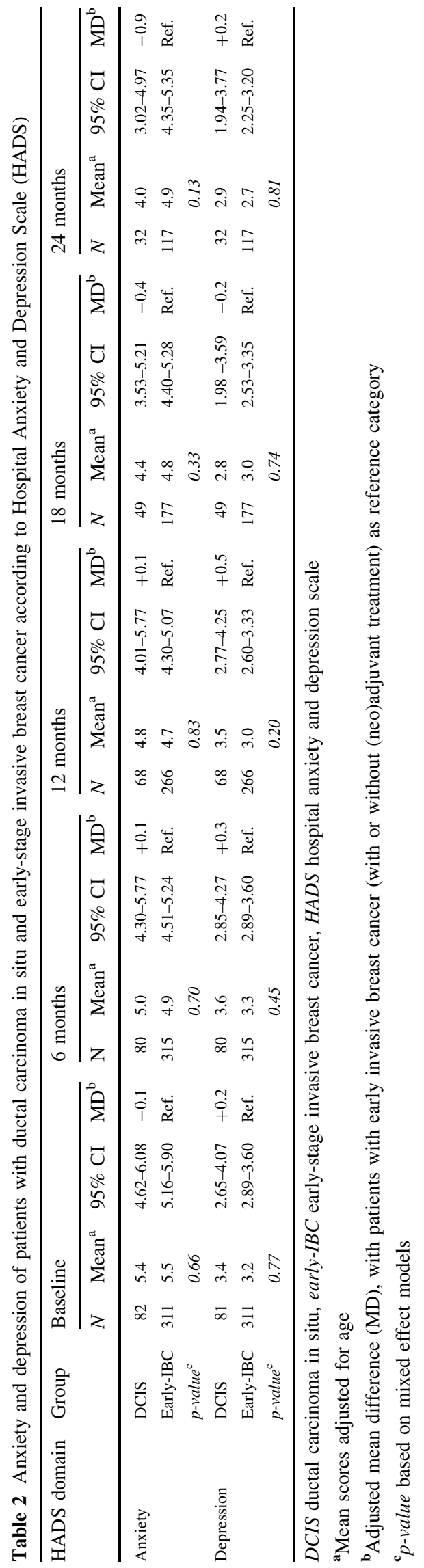

groups. Health-related QoL scores of DCIS and early-IBC patients did not differ from the reference population.

\section{Discussion}

The proportion of patients with DCIS with high-risk depression scores, indicating possible depressive disorders, was significantly higher compared to that of patients with early-IBC. Patients with DCIS still report similar levels of anxiety and HRQoL, as compared to patients with earlyIBC, despite abundant literature and online documents available to patients confirming the excellent prognosis of DCIS.

To our knowledge, this is the first study to provide prospectively collected information from a large group of DCIS and early-IBC patients being treated with modern day protocol, i.e., better imaging techniques, refined breast surgery, and more accurate radiotherapy. Furthermore, this is the only study that directly compares these two patient groups at different time intervals in the first two years after surgery. Although on average psychological distress scores were similar between DCIS and early-IBC patients, the proportion of patients with DCIS reporting high-risk depression scores was significantly higher compared to that of early-IBC patients. For both groups, high-risk anxiety symptoms scores were high at time of diagnosis and decreased over time, while the proportion of DCIS patients with high-risk depression scores increased during the first 18 months. HRQoL scores were similar between DCIS and early-IBC patients and equivalent to the Dutch reference population.

Several previous studies showed similar levels of psychological distress and HRQoL in patients with DCIS and early-IBC. A recent systematic review on 17 studies of PROs after a diagnosis of DCIS showed persistent exaggerated perceptions of the risk of breast cancer recurrence and death from breast cancer [16]. Higher perceived risk of recurrence has been associated with general anxiety and chronic anxiety [4, 16, 27]. Only two studies in breast cancer patients compared psychological morbidity (i.e., anxiety and depression) directly between DCIS and earlyIBC patients [13, 17], while HRQoL between both groups was compared in 3 studies $[12,13,15]$. In a cross-sectional study from Rakovitch et al., women with DCIS and earlyIBC treated between 1998 and 1999 expressed similar levels of anxiety and depression related to their breast cancer within the first four months after breast surgery [17]. In a Canadian prospective cohort study of 800 breast cancer patients treated in 2003, women with DCIS ( $n=107)$ had similar levels of mental health during the first year after surgery compared to women with early-IBC, irrespective of the use of adjuvant chemotherapy [13]. In 


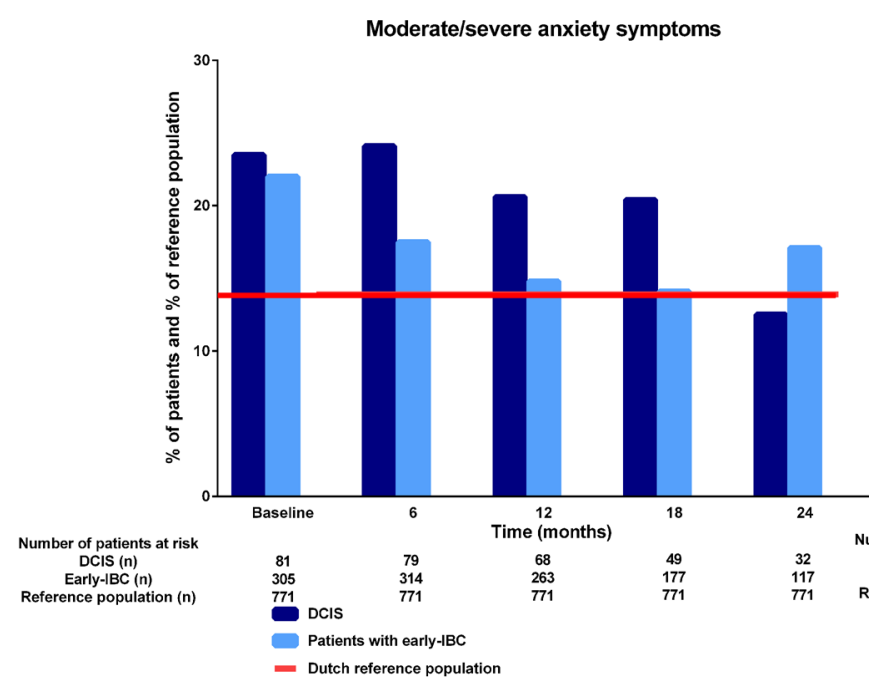

Fig. 3 Proportion of patients ductal carcinoma in situ and patients with early invasive breast cancer with or without (neo)adjuvant systemic treatment and the Dutch reference population, with high

the same study, women with DCIS and early-IBC reported similar distress levels as the reference population six months after treatment [13]. Besides anxiety and depressive symptoms, fear of breast cancer recurrence and exaggerated risk perception was similar in patients with DCIS and those with stage 1 invasive breast cancer and persisted for years after diagnosis [14, 29]. Although DCIS patients were aware of their diagnosis and significantly better able to differentiate between in situ and invasive carcinoma than women with early-IBC, they estimated their risk of recurrence of (invasive) breast disease similarly high as patients with invasive cancer $[15,30,31]$. A recent cross-sectional study from Denmark on psychological distress in DCIS patients only, reported that $20 \%$ of patients reported high anxiety scores ( $\geq 8$ HADS anxiety) years after surgery. In contrast to our results, they found a lower proportion $(6 \%)$ of patients with high depression scores ( $\geq 8$ HADS depression) 1-3 years after surgery. In the same study, the need for rehabilitation services was assessed. Unmet rehabilitation needs were reported by $29 \%$ of the patients [32]. These results indicate that there is a strong need for appropriate support and rehabilitation services even years after treatment for DCIS in this patient group. Mertz et al. did not compare DCIS patients to patients diagnosed with invasive breast cancer [32].

Previous studies on HRQoL also found similarities between DCIS and early-IBC. Longitudinal study from Jeffe et al. observed similar general health scores in the first two years after surgery between DCIS and invasive breast cancer patients (primary stage 0-2A breast cancer without neo-adjuvant chemotherapy) [15]. In a cross-sectional Dutch population-based study by van Gestel et al.,

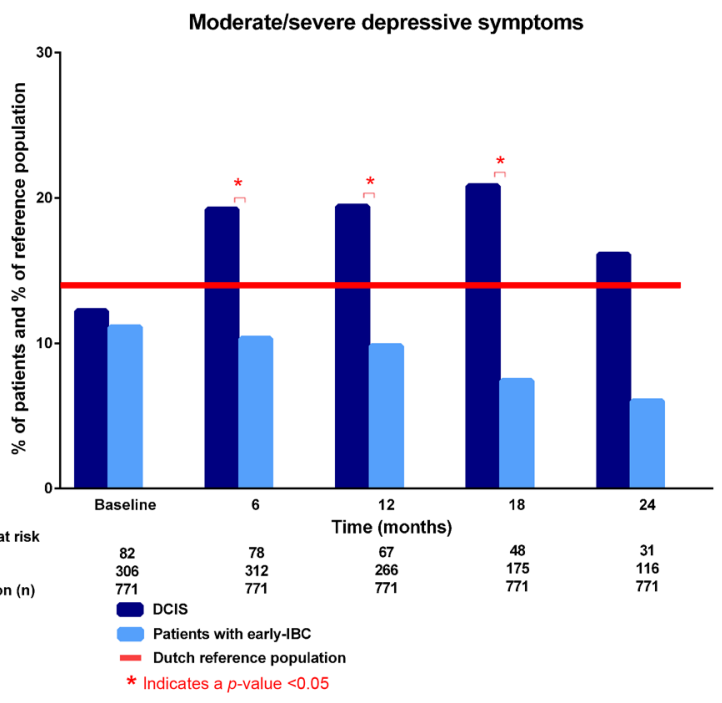

scores on the Hospital Anxiety and Depression Subscale (HADS) $(>8)$ indicating probable or presence of anxiety or depression disorders

HRQoL two to three years after diagnosis was comparable in women with DCIS and early-IBC [12]. Unexpectedly, DCIS and early-IBC patients did not only report similar HRQoL levels but these levels were also comparable to age-matched control women in both studies $[12,15]$.This can be explained by a phenomenon called response shift. The so-called response shift [33] is characterized by a change in internal standards when reporting PROs [34, 35]. In a recent longitudinal study by Hart et al. on healthrelated quality of life in DCIS patients and controls without a history of breast cancer, mental quality of life more than ten years after diagnosis of DCIS declined and was significant lower compared to levels shortly after diagnosis and compared to control group [36].This suggests that although differences are not present shortly after diagnosis, differences in HRQoL might appear years after treatment for DCIS.

Our results suggest that information on prognosis and tumor biology of DCIS that is provided to patients is still not sufficient, as DCIS patients still experience similarly high levels of anxiety as patients with invasive cancer and more often report high-risk depression scores. Nevertheless, our results also suggest that incomplete/inadequate information on prognosis may not be the main reason for patients' psychological stress, as we did not find any difference between DCIS, early-IBC treated without chemotherapy, and those treated with chemotherapy. This is counter-intuitive since those receiving chemotherapy had a more aggressive treatment required by a higher risk disease. However, studies suggest that chemotherapy is independently associated with the risk of depression and anxiety in patients with invasive breast cancer [37]. It may 
be more likely that patients experience some form of posttraumatic stress, which may be related to the diagnostic process, treatment, and the feeling of abandonment after the end of treatment, which is similar for all patients and independent of the prognostic differences. Levels of depression scores in patients with DCIS might be higher due to the fact that these patients have higher unmet needs for psychological support. It seems plausible that rehabilitation programs are offered less often to patients with DCIS compared to patients with invasive breast cancer because of the precancerous condition of DCIS, but this may need further research.

The strength of our study is that-unlike the available previously discussed historical literature-this study was performed in a prospective cohort in which PROs of DCIS patients were directly compared to early-IBC patients. In addition, it provides data on the current situation after ten years of knowledge about similar levels of anxiety and depression in patients with DCIS and early-IBC. Furthermore, it includes longitudinal data in which PROs were measured regularly within 24 months follow-up.

This study is limited by the fact that we only selected patients who were referred to the UMC Utrecht for adjuvant radiotherapy treatment and we did not include DCIS patients who underwent mastectomy, since there was no indication for adjuvant radiotherapy in these patients. This had implications to the generalizability of our results. Another limitation is that our baseline measurement took place several weeks after diagnosis (after surgery, before start of adjuvant radiotherapy). We may have missed a peak in level of anxiety around diagnosis and surgery, which might have declined somewhat in both groups as compared to the preoperative distress level.

In conclusion, the results of this study show that in recent years severe depression scores are more common in DCIS patients even months after surgery, despite their much more favorable prognosis. Anxiety levels and HRQoL are still similar in patients diagnosed with DCIS and women with early-IBC. Our results are surprising, since today, patients have better access to information on DCIS than a decade ago, and as physicians and nurses are more aware of the good prognosis of DCIS. Therefore, there is a need to further explore the cause of this distress to prevent DCIS patients from unnecessary psychological distress.

\section{Compliance with ethical standards}

Conflict of interest The authors declare that they have no conflict of interest.

Ethical approval All procedures performed in studies involving human participants were in accordance with the ethical standards of the institutional and/or national research committee and with the 1964
Helsinki Declaration and its later amendments or comparable ethical standards.

Informed consent Informed consent was obtained from all individual participants included in the study.

Open Access This article is distributed under the terms of the Creative Commons Attribution 4.0 International License (http://crea tivecommons.org/licenses/by/4.0/), which permits unrestricted use, distribution, and reproduction in any medium, provided you give appropriate credit to the original author(s) and the source, provide a link to the Creative Commons license, and indicate if changes were made.

\section{References}

1. Schwartz GF, Solin LJ, Olivotto IA, Ernster VL, Pressman PI (1999) Consensus Conference Committee (2000) The consensus conference on treatment of in situ ductal carcinoma of the breast, April 22-25. Breast J 6(1):4-13

2. Ernster VL, Ballard-Barbash R, Barlow WE, Zheng Y, Weaver DL, Cutter G et al (2002) Detection of ductal carcinoma in situ in women undergoing screening mammography. J Natl Cancer Inst 94(20):1546-1554

3. Fallowfield L, Matthews L, Francis A, Jenkins V, Rea D (2014) Low grade ductal carcinoma in situ (DCIS): how best to describe it? Breast 23(5):693-696

4. Netherlands Comprehensive Cancer Organisation (IKNL). [2017,May]. Available from: https://www.iknl.nl/over-iknl/ about-iknl or www.cijfersoverkanker.nl

5. Siegel R, J MJ, Zou Z, Jemal A (2014) Cancer statistics, 2014. CA Cancer J Clin 64(1):9-29

6. Allegra CJ, Aberle DR, Ganschow P, Hahn SM, Lee CN, Millon Underwood S et al (2010) National Institutes of health State-ofthe-Science Conference statement: diagnosis and management of ductal carcinoma in situ September 22-24. J Natl Cancer Inst 102(3):161-169

7. Narod SA, Iqbal J, Giannakeas V, Sopik V, Sun P (2015) Breast cancer mortality after a diagnosis of ductal carcinoma in situ. JAMA Oncol 20(10):888-896

8. Ernster VL, Barclay J, Kerlikowske K, Wilkie H, Ballard-Barbash R (2000) Mortality among women with ductal carcinoma in situ of the breast in the population-based surveillance, epidemiology and end results program. Arch Intern Med 160(7): 953-958

9. Saadatmand S, Bretveld R, Siesling S, Tilanus-Linthorst M (2015) Influence of tumour stage at breast cancer detection on survival in modern times: population based study in 173797 patients. BMJ 351:4901

10. Burstein HJ, Polyak K, Wong JS, Lester SC, Kaelin CM (2004) Ductal carcinoma in situ of the breast. N Engl Med 350(14): 1430-1441

11. Koch L, Jansen L, Brenner H, Arndt V (2013) Fear of recurrence and disease progression in long-term ( $>5$ years) cancer survivors -a systematic review of quantitative studies. Psycho-Oncology 22(1):1-11

12. van Gestel YRBM, Voogd AC, Vingerhoets AJJM, Mols F, Nieuwenhuijzen GAP, van Driel OJR et al (2007) A comparison of quality of life, disease impact and risk perception in women with invasive breast cancer and ductal carcinoma in situ. Eur $\mathbf{J}$ Cancer 43(3):549-556

13. Lauzier S, Maunsell E, Levesque P, Mondor M, Robert J, André $\mathrm{R}$ et al (2010) Psychological distress and physical health in the 
year after diagnosis of DCIS or invasive breast cancer. Breast Cancer Res Treat 120(3):685-691

14. Liu Y, Pérez M, Schootman M, Aft RL, Gillanders WE, Jeffe DB (2011) Correlates of fear of cancer recurrence in women with ductal carcinoma in situ and early invasive breast cancer. Breast Cancer Res Treat 130(1):165-173

15. Jeffe DB, Perez M, Liu Y, Collins KK, Aft RL, Schootman M (2012) Quality of life over time in women diagnosed with ductal carcinoma in situ, early-stage invasive breast cancer, and agematched controls. Breast Cancer Res Treat 134(1):379-391

16. King MT, Winters ZE, Olivotto IA, Spillane AJ, Chua BH, Saunders C et al (2017) Patient reported outcomes in ductal carcinoma in situ: a systematic review. Eur J Cancer 71:95-108

17. Rakovitch E, Franssen E, Kim J, Ackerman I, Pignol JP, Paszat L et al (2003) A comparison of risk perception and psychological morbidity in women with ductal carcinoma in situ and early invasive breast cancer. Breast Cancer Res Treat 77(3):285-293

18. Young-Afat DA, van Gils $\mathrm{CH}$, van den Bongard HJGD, Verkooijen HM (2017) The Utrecht cohort for multiple breast cancer intervention studies and long-term evaluation (UMBRELLA). Breast Cancer Res Treat. doi:10.1007/s10549017-4242-4

19. Netherlands Comprehensive Cancer Organisation (IKNL). [2016,May]. Available from: https://www.iknl.nl/over-iknl/ about-iknl or www.cijfersoverkanker.nl

20. van de Poll-Franse LV, Horevoorts N, van Eenbergen MDJ, Roukema JA, Aaronson NK, Vingerhoets A et al (2011) Profiles Registry G (2011) The Patient Reported Outcomes Following Initial treatment and Long term Evaluation of Survivorship registry: scope, rationale and design of an infrastructure for the study of physical and psychosocial outcomes in cancer survivors. Eur $\mathbf{J}$ Cancer 47:2188-2194

21. Zigmond AS, Snaith RP (1983) The hospital anxiety and depression scale. Acta Psychiatr Scand 67(6):361-370

22. Snaith RP (2003) The hospital anxiety and depression scale. Health Qual Life Outcomes. 1:29

23. Smith AB, Selby PJ, Velikova G, Stark D, Wright EP, Gould A et al (2002) Factor analysis of the hospital anxiety and depression scale from a large cancer population. Psychol Psychother 75(3):165-176

24. Aaronson NK, Ahmedzai S, Bergman B, Bullinger M, Cull A, Duez NJ et al (1993) The European organization for research and treatment of cancer QLQ-C30: a quality-of-life instrument for use in international clinical trials in oncology. J Natl Cancer Inst 85(5):365-376

25. Fayers PM, Aaronson NK, Bjordal K, Groenvold M, Curran D, Bottomly A (2001) The EORTC QLQ-C30 Scoring Manual, 3rd edn. European Organisation for Research and Treatment of Cancer, Brussels

26. Bonnetain F, Fiteni F, Efficace F, Anota A (2016) Statistical challenges in the analysis of health related quality of life in cancer clinical trials. J Clin Oncol 34(16):1953-1956

27. Partridge A, Adloff K, Blood E, Dees EC, Kaelin C, Golshan M et al (2008) Risk perceptions and psychological outcomes of women with ductal carcinoma in situ: longitudinal results from a cohort study. J Natl Cancer Inst 100:243-251

28. Rothrock NE, Matthews AK, Sellergren SA, Flemming G, List M (2004) State anxiety and cancer-specific anxiety in survivors of breast cancer. J Psychosoc Oncol 22:93-109

29. Liu Y, Pérez M, Schootman M, Aft RL, Gillanders WE, Ellis MJ et al (2010) A longitudinal study of factors associated with perceived risk of recurrence in women with ductal carcinoma in situ and early-stage invasive breast cancer. Breast Cancer Res Treat 124(3):835-844

30. Liu Y, Pérez M, Aft RL, Massman K, Robinson E, Myles S et al (2010) Accuracy of perceived risk of recurrence among patients with early-stage breast cancer. Cancer Epidemiol Biomarkers Prev 19(3):675-680

31. Virnig BA, Tuttle TM, Shamliyan T, Kane RL (2010) Ductal carcinoma in situ of the breast: a systematic review of incidence, treatment and outcomes. J Natl Cancer Inst 102(3):170-178

32. Mertz BG, Duriaud HM, Kroman N, Andersen KG (2017) Pain, sensory disturbances and psychological distress are common sequelae after treatment of ductal carcinoma in situ. Acta Oncol 56(5):724-729

33. Rees J, Clarke MG, Waldron D, OBoyle C, Ewings P, MacDonagh RP (2005) The measurement of response shift in patients with advanced prostate cancer and their partners. Health Qual Life Outcomes 3:21

34. Schwartz CE, Finkelstein JA, Rapkin BD (2017) Appraisal assessment in patient-reported outcome research: methods for uncovering the personal context and meaning of quality of life. Qual Life Res 26(3):545-554

35. Sprangers MA, Schwartz CE (1999) Integrating response shift into health-related quality of life research: a theoretical model. Soc Sci Med 48(11):1507-1515

36. Hart V, Sprague BL, Hampton JM, Newcomb PA, Gangnon RE, Trentham-Dietz A (2016) Trends in health-related quality of life after a diagnosis of ductal carcinoma in situ. J Clin Oncol 34(12):1323-1329

37. Yang H, Brand JS, Fang F, Chiesa F, Johansson ALV, Hall P et al (2017) Time-dependent risk of depression, anxiety and stressrelated disorders in patients with invasive and in situ breast cancer. Int J Cancer 140(4):841-852 